of Yale University, and particularly of the members of the mathematical department were gratefully acknowledged by a unanimous vote of thanks and appreciation at the closing meeting.

Detailed reports of the courses, prepared by the lecturers, will appear in later numbers of the BuLlETIN.

Virgil SNyder.

\title{
THEORY AND CONSTRUCTION OF TABLES FOR THE RAPID DETERMINATION OF THE PRIME FACTORS OF A NUMBER.*
}

BY PROFESSOR ERNEST LEBON.

By making use of some hitherto unnoticed properties of certain arithmetic progressions, I have succeeded in constructing a table giving very rapidly the solution of the following double problem : To determine whether a given number is prime or composite, and in the latter case to find its prime factors. The process which I employ is applicable to large numbers. $†$

1. Let $B$ be the product $\alpha \beta \ldots \lambda$ of the consecutive prime numbers $\alpha, \beta, \ldots, \lambda$, beginning with $2 ; P$ the product $(\alpha-1)(\beta-1) \cdots(\lambda-1) ; I$ any of the $P$ numbers that are relatively prime to $B$ and less than $B ; K$ a number successively equal to the positive integers, starting from zero.

We easily see that the system of $P$ arithmetic progressions whose general term is $B K+I$ contains all the prime numbers except those that occur in $B$.

We shall say that $B$ is the base of the system and that $I$ is the index of a term of this system.

Two indices will be said to be complementary when their sum is equal to the base.

2. Let $N, D$ and $M$ be any numbers relatively prime to $B$. In order to avoid ambiguity, I will write $D$ in the form $B K^{\prime}+I^{\prime}$.

It is evident that $N(=B K+I)$ is or is not divisible by $D$ according as $K$ and $M$ do or do not satisfy the equation

* Translated by Professor W. B. Fite.

† CH. Comptes rendus, vol. 151 (1905), p. 78. See also \& 10, p. 77. 


$$
B K+I=M D,
$$

$B, I$ and $D$ being known.

3. Let $k$ and $m$ be the minimum values of $K$ and $M$ satisfying equation (a), and $n$ a number successively equal to the positive integers starting from zero. If necessary for clearness, I use $k_{I}$ for the numbers $K$ relative to a divisor $D$.

The equality

$$
K=k+n D
$$

gives the values of $K$ to which correspond all the numbers $N$ that are divisible by $D$.

From this equality we get the formula

$$
n=\frac{K-k}{D}
$$

where $K$ is the integral quotient obtained by dividing $N$ by $B$; the remainder in this division is the value of $I$.

We see that according as the value of $n$ obtained by applying formula (1) is integral or fractional, the number $N$ is, or is not, a multiple of the divisor $D$.

Then the table of numbers $k$ set up for a system of base $B$ enables one to recognize whether $N$ is prime or not by dividing the difference $K-k$ by the prime numbers less than $\sqrt{N}$ and greater than $\lambda$; if $N$ is not prime, this procedure gives its prime factors.

We see that the larger the base $B$ the more rapidly this method gives the result.

Before applying formula (1), it should not be forgotten that if we are considering a number $N^{\prime}$. we must in order to get $N$ remove from it the factors that are common to it and $B$.

4. The numbers $k$ I shall call characteristics.

5. In order to find methodically and quickly the characteristics $k$ which correspond to the $P$ arithmetic progressions of a system with the base $B$, we can use the following formula, which is obtained by replacing in equation $(a) K$ and $M$ by $k$ and $m$, and $D$ by $B K^{\prime}+I^{\prime}$ :

$$
k=\frac{I^{\prime} m-I}{B}+K^{\prime} m .
$$


Formula (2) gives the characteristic $k$ when the value of $m$ is such that the binomial $I^{\prime} m-I$ is divisible by $B$.

6 . The three following theorems, which are easily demonstrated, enable one to make a considerable reduction in the number of operations required for the calculation of the characteristics $k$ :

I. To the product $I^{\prime} m$ of the two indices $I^{\prime}$ and $m$ correspond an index $I$ and a characteristic $k$; this characteristic is associated with the number $I^{\prime} m$ by the arithmetic progression of base $B$ and index I given by this product.

II. The $P$ arithmetic progressions of a system of base $B$ being arranged in the order of the increasing values of the indices $I$ of their terms, the sum of the two characteristics $k$ and that of the two values of $m$ relative to the same divisor $D$ and to two progressions equidistant from the extremes are equal to $D-1$ and $B$ respectively.

III. If the values of $I, k_{I}, I^{\prime}$, and $m$ satisfy the equation

$$
B k_{I}+I=I^{\prime} m,
$$

and if we consider the equation

$$
B k_{B-I}+(B-I)=\left(B-I^{\prime}\right) m,
$$

where the two indices $B-I$ and $B-I^{\prime}$ are complementary to the $I$ and $I^{\prime}$ respectively of the preceding equality, the unknown characteristic $k_{B-I}$ is given by the formula

$$
k_{B-I}=m-1-k_{I} .
$$

7. It follows from Theorems II and III that in order to calculate the binomial $I^{\prime} m-I$, it is sufficient to associate with the first half of the $P$ values of $I^{\prime}$ the first half of the $P$ values of $m$, arranged in the order of magnitude.

The remainder obtained by dividing $I^{\prime} m$ by $B$ is the index $I$ relative to a progression of the system of base $B$.

When $K^{\prime}$ is zero, the first term of formula (2) gives, in each of the $P$ progressions of base $B$, the $P$ characteristics $k$ corresponding to the $P$ values of $I$.

Inasmuch as the characteristics $k$ corresponding to the index $I$ are the same when $D$ is equal to either $I^{\prime}$ or $m$, it follows from Theorem $I$ that it is sufficient to take the products $I^{\prime} m$ 
starting from the value of $m$ equal to the value of $I^{\prime}$; that is to say, it is sufficient to take the values of $I^{\prime} m$ starting from $I^{\prime 2}$. We know that we apply the first term of formula (2) only to the values of $m$ which are equal to the first $P / 2$ indices. Moreover to the products of 1 by the indices correspond characteristics $k$ which are evidently zero.

Consequently, among the $P^{2}$ characteristics $k$ relative to the $P$ divisors which equal the indices there are at most $P(P-2) / 8$ characteristics whose determination requires a multiplication and a division.

8. As to the $P$ characteristics $k$ relative to a divisor $D$ superior to $B-1$ and with index $I^{\prime}$, we can deduce them immediately from the $P$ characteristics found for $D=I^{\prime}$ by making use of the last term of formula (2).

9. In order to apply formula (1), we can make use of a table of characteristics relative to the base $B$ containing at the top of the columns only the first half of the $P$ indices $I$ arranged in order of magnitude, and below each index $I_{n}$ the complementary index $B-I_{n}$; then in these columns, in regard to the prime divisors $D$, the values of $k$ relative to the first half of the $P$ indices $I$.

Then, having a number $N$ which does not contain any of the prime factors of $B$, we divide $N$ by $B$. This gives the quotient $K$ and the remainder $I$, which $I$ shall call $I_{n}$ if it belongs to the first half of the $P$ indices $I$ arranged in order of magnitude, and $I_{n^{\prime}}$ if it belongs to the second half of these indices.

When the remainder is $I_{n}$, the index is also $I_{n}$ and the characteristic $k$ is equal to the value $k_{n}$ given in the table.

According as $D$ is, or is not, a multiple of the difference $K-k_{n}, D$ is, or is not, a prime divisor of the number whose index is $I_{n}$ or of the number whose index is $I_{n^{\prime}}$.

10. The table of characteristics relative to the base 30030 , with the prime divisors from 17 to 30029 enables one to solve the problem in question between 1 and $30030^{2}$ or 901800900 .

Suppose that the table of characteristics $\dot{k}$ relative to the base $B$ is formed of columns headed by all the indices $I$ in order of magnitude and of rows headed by the prime divisors $D$ arranged in the order of magnitude. The characteristic $k$ corresponding to a number $N$ of index $I_{n}$ and to a prime divisor $D$ is found at the intersection of the column $I_{n}$ and the row $D$.

Let $N$ be a number of the form $30030 K+I$. In making 
the trial we will stop at the prime divisor $D_{n}$ immediately inferior to $\sqrt{N}$.

We consider whether $K$ is equal to one of the characteristics which correspond to the index $I$; for this it is sufficient to start from the prime divisor immediately superior to $K$.

When $K$ is equal to one or several of these characteristics, $N$ admits prime divisors which correspond to these characteristics. Then we have immediately the composition of $N$.

When $K$ is not equal to any of these characteristics, we form the differences $K-k$ for the prime divisors $17,19,23, \ldots$. These differences are always less than 30029 , because $K$ is here less than $B$ and $k$ is less than $D$ and hence less than $B$. A difference $K-k$ is, or is not, equal to an index. In the former case, we recognize without calculation whether the difference $K-k$ is divisible by the divisor that corresponds to it. In the latter case we recognize nearly always whether a difference $K-k$ is divisible by the corresponding divisor $D$ without performing the division; then we decompose $K-k$ into factors, one of which is either one of the prime numbers $2,3,5,7,11$ and 13 , or a product of some of these, and the other an index. In most cases it is not necessary to perform this decomposition in order to see if a difference is divisible by the prime divisor which corresponds to it.

If there is no difference $K-k$ that is divisible by any of the prime factors from 17 to $D_{n}, N$ is prime. If we find a difference $K-k$ that is divisible by the prime divisor $D$ less than $D_{n}, N$ is divisible by $D$. We divide $N$ by $D$, the resulting quotient also by $D$, and so on. Let $N_{1}$ be the last quotient thus obtained. We treat $N_{1}$ as we have just treated $N$, beginning with the prime divisor immediately following $D$, and we find that $N_{1}$ is the product of characteristics or is prime.

11. In order to recognize instantly whether a difference $K-k$ is divisible by the corresponding divisor $D$, it is sufficient to have, in addition to the table of characteristics relative to the base 30030 up to the divisor 30029 , a table of remainders obtained by dividing the consecutive integers from 17 to 30029 by the divisors $D$; in fact, a difference $K-k$ is divisible by the corresponding divisor $D$, when the values of $R$ and of $k$ which correspond to this division are equal.

PARIS,

May, 1906. 